\title{
Classification of empirically derived prototypes as a function of category experience
}

\author{
TIMOTHY J. BREEN and ROGER W. SCHVANEVELDT \\ New Mexico State University, Las Cruces, New Mexico
}

In three experiments, subjects learned to classify dot patterns into three categories represented by either three, six, or nine exemplars. Following learning, subjects were tested on an additional set of patterns, which included patterns from the learning phase, the category objective prototypes, and new distortions of the objective prototypes. Also included in the test set were empirical prototypes and distortions of empirical prototypes. Empirical prototypes were derived by averaging feature values of category exemplars in the learning phase. The overall results revealed that empirical prototypes were classified more accurately than were objective prototypes. In addition, a pattern of convergence in error rates was observed for distortions of the objective and empirical prototypes as category set size increased, but this same pattern was not observed for the objective and empirical prototypes themselves. This lack of convergence for the prototypes is inconsistent with explanations of the category set size effect that rely on the central limit theorem.

In a common paradigm for investigating categorization processes, subjects learn to classify objects into two or more categories (learning phase), and then are tested on an additional set of patterns (transfer phase). Characteristics of the learning and test patterns are manipulated in order to uncover the processes involved. For example, researchers have investigated the effects of the distance from test patterns to the category center (Posner \& Keele, 1968), the time elapsed between the learning and transfer phases (Posner \& Keele, 1970), and the similarity between test patterns and learning patterns (Homa, Sterling, \& Trepel, 1981; Medin \& Schaffer, 1978).

A robust finding in classification research is that increases in the number of exemplars defining a category during the learning phase lead to more accurate classification in the transfer phase (Homa \& Chambliss, 1975; Homa, Cross, Cornell, Goldman, \& Schwartz, 1973; Homa et al., 1981). This result has been labeled the category set size effect. Most, if not all, studies investigating the category set size effect have used a common procedure for generating a category. This procedure involves first randomly constructing a template or objective prototype pattern, and then applying a statistical distortion rule to generate additional category members.

An example of this kind of procedure is the construction of dot-pattern categories (Posner, Goldsmith, \& Welton, 1967). To generate a category of dot patterns, an objective prototype is first created by randomly assigning dots into cells of a matrix. To generate additional dot patterns from the same category, a rule that is applied to the

The authors thank Nancy Cooke, Don Dearholt, and Frank Durso for comments on earlier drafts of this manuscript. Thanks also to Mel Tempel and Robin Smith for assistance in collecting the data. Requests for reprints should be directed to Timothy J. Breen, Department of Psyshology, Box 3452, New Mexico State University, Las Cruces, NM 38003 . objective prototype pattern moves the dots to a new position in the matrix. A rule can be selected such that on the average, it will move dots relatively far away from their original position (high distortions) or relatively close to their original position (low distortions). Several experiments have shown that classification accuracy of novel patterns in the transfer phase is a function of the degree of similarity between the pattern and its objective prototype (e.g., Homa \& Vosburgh, 1976; Posner \& Keele, 1970; Strange, Kenney, Kessel, \& Jenkins, 1970). That is, objective prototypes are classified more accurately than are distortions of objective prototypes, and low distortions are classified more accurately than are high distortions.

The motivation for the following experiments was to evaluate the consequences of employing such procedures in experiments designed to investigate category set size effects. The subjects' task was to learn to classify $18 \mathrm{dot}$ patterns into three categories. A category was represented by either three, six, or nine high-level distortions (patterns that are relatively distant from the category prototype) in the training set. Following the learning phase, subjects were asked to classify old patterns from the training set and new patterns that they had not seen before.

The novel feature of these experiments is the use of empirical prototypes. Empirical prototypes are patterns derived by averaging features of the training patterns from a category. Thus, these are stimulus patterns that should reflect the true center of a category to a greater extent than would the randomly determined objective prototype. To determine an empirical prototype, an average $(x, y)$ coordinate value is determined for each dot (feature) for patterns from a single category in the training set. Therefore, an empirical prototype can be generated for each of three categories consisting of three, six, or nine exemplars. Patterns classified during the transfer phase con- 
sisted of old patterns, the objective prototypes, the empirical prototypes, and patterns generated by distorting the empirical and objective prototypes. New distortions included both low-level distortions and high-level distortions of the empirical and objective prototypes.

We expected that classification accuracy for empirical prototypes would show an advantage over objective prototypes, especially when a category was represented by only three exemplars during learning. However, we expected that this advantage would decrease as category set size increased. We predicted this interaction because we thought that the objective and empirical prototypes would converge as category set size increased. This convergence follows from the central limit theorem, by assuming that the objective prototype corresponds to the population mean and that the empirical prototype corresponds to the sample mean of $n$ (category set size) cases. In addition, if the objective and empirical prototypes converge across category set size, it follows that distortions of the objective and empirical prototypes should converge across category set size. For this reason, we also expected an interaction in classification performance on distortions of the objective and empirical prototypes.

An important goal in these experiments was to observe subjects' responses to changing statistical properties of category exemplars in relation to category size. Previous research investigating related issues has employed a relatively limited sample of stimuli within a particular stimulus domain. In the present experiments, unique patterns and categories were generated for each subject, so that any observations made about the statistical properties of dot-pattern categories, and any conclusion drawn from the data, would be as general as possible within the present stimulus domain.

\section{EXPERIMENT 1}

\section{Method}

Subjects. A total of 54 subjects, enrolled in an introductory psychology class at New Mexico State University, participated in the experiment in partial fulfillment of a course requirement.

Materials and Apparatus. The stimuli were dot-pattern categories generated by procedures described in detail by Posner et al. (1967). Briefly, to generate a category, a $30 \times 30$ matrix is defined and nine dots are assigned randomly into cells of the matrix. This pattern becomes the objective prototype of the category. To generate a distortion of the objective prototype, a $20 \times 20$ matrix is defined around each dot in the objective prototype and is subdivided into five areas; the objective prototype dot occupies the center area (Area 1) of the matrix. Areas 2 through 5 form concentric squares around Area 1; Area 5 occupies the outermost region in the $20 \times 20$ matrix. A probabilistic rule is then applied that selects one of the five areas for the new location of the dot (in the event that Area 1 is selected, the dot remains in its original position). Within the selected area, a cell is randomly selected as the new location of the dot. The rule is applied iteratively to all nine dots in the objective prototype pattern to form a distortion of the objective prototype. The probability that a dot will move into one of the outer areas of the matrix is greater for high distortions than for low distortions. For 3-bit distortions, the probabilities of each dot's moving into the five areas are $.59, .20, .16, .03$, and .02 , for Areas
1 through 5, respectively. For 7.7-bit distortions, the associated probabilities for Areas 1 through 5 are (in order): .00, .40, .32, .15 , and .13. Training sets consisted of 18 7.7-bit distortions from three categories. Nine patterns formed one category, 6 patterns formed a second, and 3 patterns formed the third.

After the generation of the training sets, the empirical prototype was generated by averaging the coordinates of corresponding dots for training patterns in each category. Once the empirical prototype was computed, the above procedure was performed on the empirical prototype to generate distortions of the empirical prototype.

The transfer set contained 3 patterns from the training set from each category (old patterns), the objective and empirical prototypes of each category, three 7.7-bit distortions and three 3-bit distortions of the objective prototype of each category, and three 7.7-bit and three 3-bit distortions of the empirical prototype of each category, for a total of 51 patterns.

All stimulus generation and experimental control procedures were programmed in Pascal and run on a Terak 8510 microcomputer.

Procedure. Subjects were tested individually. They were seated in front of a computer terminal, and instructions were displayed on the screen. The instructions informed subjects that the task involved learning to classify dot patterns into three different categories, labeled A, B, and C. They first saw each pattern displayed on the screen for a few seconds, with the label of the category above it. After presentation of all of the patterns and their category labels, the second part of the experiment began. Only the patterns were displayed, and subjects were required to supply the correct category label for each pattern.

During the learning phase, the order of presentation was randomized within each block of trials for every subject. If the subject made a correct response, the pattern was blanked from the screen, and the word "CORRECT" appeared in the lower center. If the subject made an incorrect response, the pattern remained on the screen and an error message appeared in the upper left-hand corner to correctly identify the category of the pattern. The misclassified pattern and the error message stayed on the screen until the subject pressed the return key, which blanked the screen and initiated the next trial. After each block of trials, the next block number and the number of errors made on the previous block of trials was displayed on the screen. The subject initiated each block of trials by pressing the return key.

The learning phase of the experiment was terminated when the subject had correctly classified all training patterns during a block of trials. If a subject did not reach the learning criterion after 30 blocks of trials, the experiment was terminated. After the subject had reached criterion learning, another set of instructions was presented on the screen:

In this final part of the experiment, you will again be asked to classify patterns from the same three categories. Most of these patterns, however, you have not seen before. As you probably noticed, in the last part of the experiment, each category had a different number of members. This will not be the case in this next part: one third of the patterns will be from Category A, one third from Category B, and one third from Category $C$. There will be 17 pattems from each category, for a total of 51 .

Unlike the last part of the experiment, you will only have to classify each pattern once, so that if you make an error, you won't have to go back through all the patterns again. Please ask the experimenter if you have any questions.

The transfer phase was then initiated after the subject pressed the space bar. On each trial during the transfer phase, the pattern was blanked from the screen after the subject made his/her response, and correct/incorrect feedback was given. The order of presentation of transfer patterns was also randomized for each subject.

All stimuli were generated independently for each subject prior to the experimental session. That is, each subject saw a different 
set of categories and patterns. The category labels A, B, and C referred with approximately equal frequency to categories represented in the learning phase by three, six, and nine exemplars.

\section{Statistical Properties of the Stimuli}

To clarify the convergence trends between the objective and empirical prototypes and between distortions of these patterns, we generated 1,000 stimulus sets and calculated the distances shown in Table 1 . The distance between two patterns was calculated by summing the euclidean distances between corresponding dots in the patterns. Table 1 shows that the distance between the objective and empirical prototype decreases across category set size, and the distance between the distortions of the empirical and objective prototype converges across category size.

This convergence can also be described in terms of distances to the most similar old pattern also shown in Table 1. At a category set size of three, the distance between the empirical prototype and the closest old pattern is less than the distance between the objective prototype and its closest old pattern. However, this difference disappears as category set size increases. This same kind of interaction occurs with distortions of the empirical and objective prototypes.

\section{Results and Discussion}

Of the 54 subjects participating in the experiment, 9 failed to meet the learning criterion of one perfect classification block, and these data were excluded from the analyses. The mean number of blocks to the learning criterion for the remaining subjects was 11.5 .

Percent errors for patterns in the transfer set are shown in Figures 1 and 2. An analysis of variance was performed for classification performance on the empirical and objective prototypes, treating prototype as a factor with two levels (empirical and objective), and category size as a factor with three levels (three, six, and nine), resulting in a $(2 \times 3)$ factorial design. The decrease in errors with the number of exemplars in the training set was significant $[F(2,88)=3.74, M S e=1,257, p<.05]$. Classification of objective prototypes was significantly less ac-

Table 1

Mean Distances Between Test Set Patterns to the Empirical Prototypes and the Most Similar Old Pattern

\begin{tabular}{clrrrrrc}
\hline \multirow{2}{*}{$\begin{array}{c}\text { Category } \\
\text { Size }\end{array}$} & & \multicolumn{7}{c}{ Test Pattern } \\
\cline { 3 - 8 } 3 & NLO & NHO & NLE & NHE & OP & EP \\
\hline \multirow{3}{*}{6} & 33.05 & 48.63 & 29.99 & 45.08 & 30.56 & 28.05 \\
& EP & 26.55 & 46.00 & 7.53 & 37.93 & 22.86 & 0 \\
& MSO & 29.44 & 45.26 & 29.03 & 43.68 & 27.16 & 26.90 \\
& EP & 20.59 & 42.50 & 7.53 & 37.90 & 17.02 & 0 \\
9 & MSO & 27.59 & 43.15 & 27.67 & 42.88 & 25.22 & 25.44 \\
& EP & 17.79 & 41.22 & 7.89 & 38.29 & 14.70 & 0 \\
\hline
\end{tabular}

Note-EP = empirical prototypes; $M S O=$ most similar old pattern; $\mathrm{NLO}=$ new low distortions of the objective prototype; $\mathrm{NHO}=$ new high distortions of the objective prototype; NLE = new low distortions of the empirical prototype; $\mathrm{NHE}=$ new high distortions of the empirical prototype; $\mathrm{OP}=$ objective prototype.

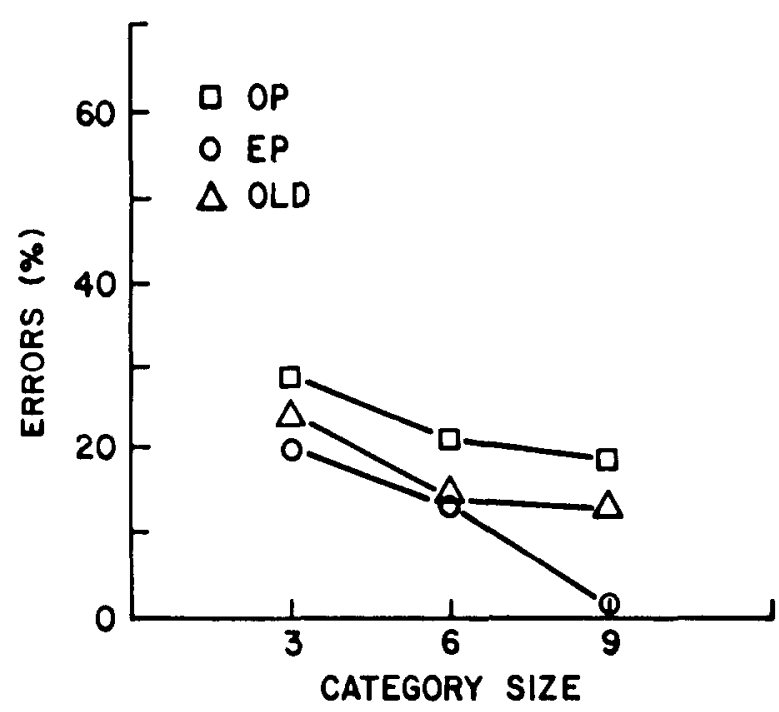

Figure 1. Percent errors for prototype and old patterns in Experiment 1.

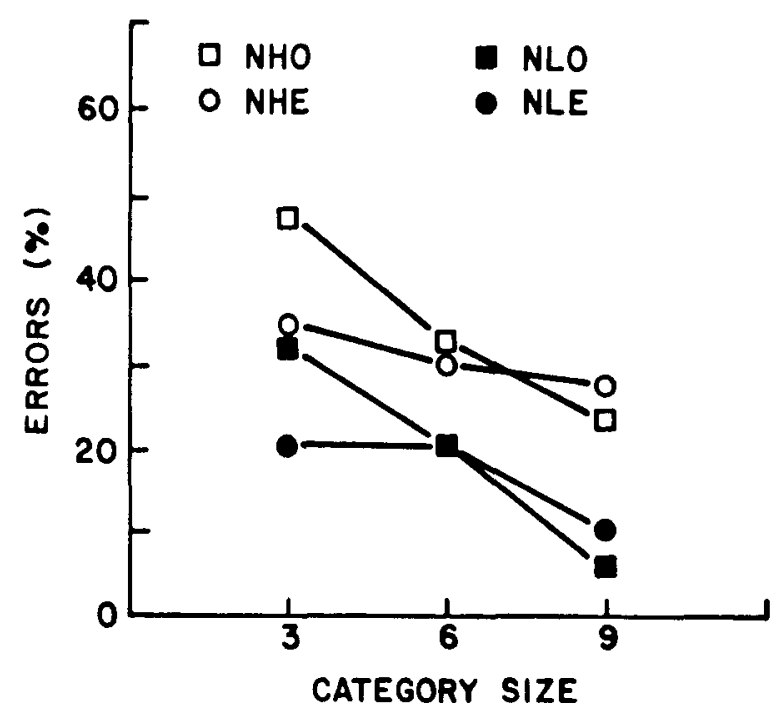

Figure 2. Percent errors for new distortions in Experiment 1.

curate than classification of the empirical prototypes $[F(1,44)=7.33, M S \mathrm{e}=1,136, p<.01]$. However, contrary to our expectations, there was no prototype $x$ category size interaction $[F(2,88)=0.34, M S e=977]$, suggesting that classification of empirical prototypes benefited from additional exemplars to the same extent that classification of objective prototypes benefited.

A second analysis of variance was performed on classification performance on new distorted patterns. In this analysis, distortion level was treated as a factor with two levels (high and low); the prototype from which new patterns were derived was treated as a second factor with two levels (empirical and objective); and category size was treated as a third factor with three levels (three, six, and nine $)$, resulting in a $(2 \times 2 \times 3)$ factorial design. The main effect of category size was again significant $[F(2,88)$ 
$=12.00, \mathrm{MSe}=1,051, p<.001]$. Also, classifications involving high distortions were less accurate than classifications of low distortions $[F(1,44)=33.46$, $M S \mathrm{e}=808, p<.001]$. The main effect of prototype approached significance $[F(1,44)=2.95, M S e=432$, $p<.10$ ], and there was a significant prototype $\times$ category size interaction $[F(2,88)=5.91, M S e=526$, $p<.005]$. This interaction confirmed our expectation that error rates would merge for distortions of objective and empirical prototypes across category set size. Fewer errors occurred on distortions of the empirical prototype at a category set size of three, but with increased exemplar experience this difference disappeared.

An analysis performed on classification of old patterns revealed that errors for old patterns decreased with category size $[F(2,88)=3.23, M S e=983, p<.05]$.

The results are consistent with previous findings that more experience in classifying different category exemplars in the learning phase is accompanied by more accurate classification of novel patterns during the transfer phase, and that low distortions are classified more accurately than high distortions. In addition, our prediction that error rates on distortions of the objective and empirical prototypes would interact across category set size was confirmed. However, the lack of interaction between the objective and empirical prototypes across category set size is inconsistent with explanations resorting to the central limit theorem. The low error rates for empirical prototypes were also surprising, especially when considering that at a category set size of 9 , the $2 \%$ error rate represents that only 1 subject misclassified the empirical prototype.

Experiment 2 was essentially a replication of Experiment 1, except that the conditions of the transfer phase were changed. Two characteristics of the transfer phase in Experiment 1 may make the interpretation of the results difficult. During the transfer phase in Experiment 1, subjects were provided correct/incorrect feedback. In contrast, in most experiments in which the subject receives an immediate transfer test, feedback is not provided. This controls to some extent the amount of learning that occurs in the transfer phase. In addition, the length of the transfer phase in Experiment 1 may have resulted in some forgetting of old patterns. Forgetting of old patterns may have resulted in two novel findings. The first was less accurate classification of old patterns than of empirical prototypes. Although previous research has not compared classification performance for old and empirical prototype patterns, old patterns are usually classified more accurately than are novel patterns in an immediate transfer test. However, in a delayed test, objective prototypes begin to show an advantage over old patterns (see Hintzman \& Ludlam, 1980, for a simulation of this effect). In addition, a decrease in errors for old patterns with category set size would be expected if forgetting of old patterns had occurred. Presumably, classification of forgotten old exemplars would then be influenced by category set size in the same way as new exemplars.
In order to make the clearest possible interpretations of subjects' classification performance during an immediate transfer test, it is optimal to reduce as much as possible the amount of learning that occurs during the transfer phase and the amount of forgetting of information acquired during the learning phase. Experiment 2 was conducted in order to reduce these possibilities. For Experiment 2, subjects received no feedback during the transfer phase, and the length of the transfer phase was reduced by decreasing the total number of patterns in the transfer set from 51 to 21 .

\section{EXPERIMENT 2}

\section{Method}

Subjects. A total of 129 subjects enrolled in an introductory psychology course at New Mexico State University participated in the experiment and received partial credit toward a course requirement.

Materials and Apparatus. All stimuli were generated independently for each subject exactly as described in Experiment 1. Training sets consisted of 18 patterns: 9 high distortions of one prototype, 6 high distortions of a second prototype, and 3 high distortions of a third prototype. Transfer sets included 21 patterns, 7 from each category. From each category there was 1 old pattern, 1 new high distortion of the empirical prototype, 1 new high distortion of the objective prototype, 1 new low distortion of the empirical prototype, 1 new low distortion of the objective prototype, the objective prototype, and the empirical prototype. The experiment was run on a Terak 8510 microcomputer.

Procedure. Experimental procedures were similar to those described in Experiment 1. During the learning phase, subjects were required to meet the learning criterion of perfect classification of all patterns during one block of trials. During learning, subjects were told whether they had correctly or incorrectly classified the previous pattern. If they misclassified a pattern, the pattern remained on the screen, and the correct category label was provided. The transfer phase was initiated once the learning criterion was reached, and the experiment was terminated if this criterion was not reached after 35 blocks of trials. The number of allowable blocks was increased over Experiment 1 in an effort to reduce the amount of discarded data. No feedback was provided during the transfer phase.

\section{Results and Discussion}

Of the 129 subjects participating in the experiment, 25 failed to meet the learning criterion of perfect classification during one block of trials, and these subjects were excluded from the analyses. For the remaining subjects, the mean number of blocks to the learning criterion was 16.7 .

Mean classification errors for prototypes and old patterns are shown in Figure 3. A $2 \times 3$ analysis of variance was performed on classification data for the prototypes, treating prototype (empirical and objective) and category set size (three, six, and nine) as factors. The decrease in errors with the number of exemplars defining a category during learning was highly significant $[F(2,208)=19.46$, $M S e=1,863, p<.001]$. The classification of objective prototypes was less accurate than was the classification of empirical prototypes $[F(1,104)=6.53, M S e=1,176$, $p<.025]$. Category size did not interact with prototype 
$[F(2,208)=0.20, M S e=1,248]$. This pattern of results mirrors the results of Experiment 1.

A second analysis of variance was performed on classification errors for new distorted patterns, which are shown in Figure 4. In this analysis, distortion level was treated as a factor with two levels (high and low), the prototype from which new patterns were derived was a second factor with two levels (empirical and objective), and category size was the third factor containing three levels (three, six, and nine). As in Experiment 1, errors for new distorted patterns decreased with category size $[F(2,208)$ $=40.62, M S \mathrm{e}=2,397, p<.001]$, and classifications of high distortions were significantly less accurate than classifications of low distortions $[F(1,104)=23.01$, $M S \mathrm{e}=2,040, p<.001]$. The main effect of prototype approached significance $[F(1,104)=3.67, M S \mathrm{e}=1,819$, $p<.10$ ]. However, the interaction between prototype and category size observed in Experiment 1 was not significant $[F(2,208)=0.48, M S \mathrm{e}=1,659]$. Figure 4 shows that this null result is caused by the lack of convergence for low empirical and objective distortions as category set sizes increased. Specifically, error rates for low distortions of the empirical and objective prototype are nearly identical at a category set size of three. A clear pattern of convergence can be seen for high distortions of the empirical and objective prototype. No other interactions between factors were significant.

Classification of old patterns was not significantly influenced by category size $[F(2,208)=2.28$, $M S \mathrm{e}=1,576, p>.10]$. The facts that error rates for empirical prototypes did not fall below error rates for old patterns and that old pattern error rates were relatively immune to changes in category size suggest that our attempt to reduce the amount of learning of new patterns and forgetting of old patterns in Experiment 1 was successful.

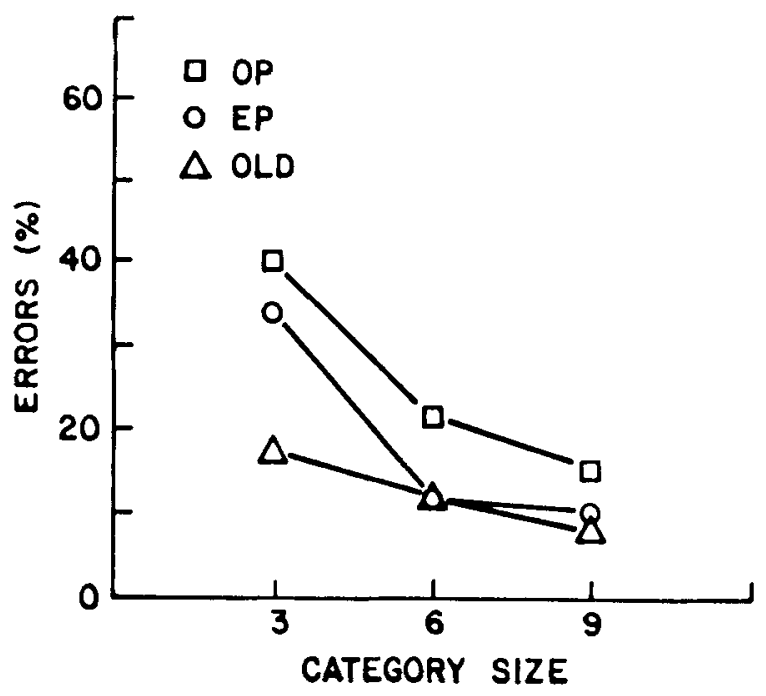

Figure 3. Percent errors for prototype and old patterns in Experiment 2.

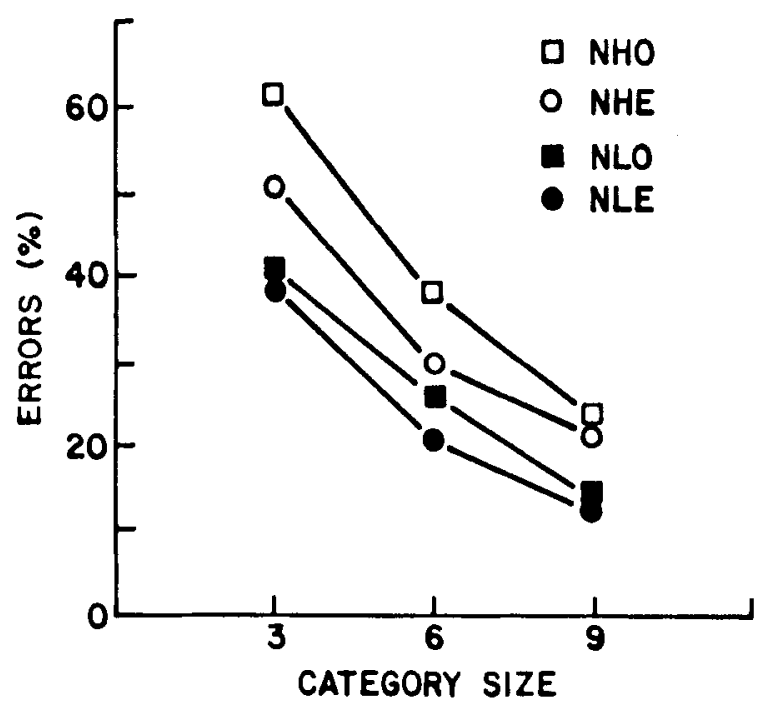

Figure 4. Percent errors of new distortions in Experiment 2.

With the exception of the failure of finding an interaction between error rates for distortions of the objective and empirical prototypes across category set size, the results are consistent with the results of Experiment 1 . The most surprising result in both experiments is that error rates for empirical prototypes remain consistently superior to error rates for objective prototypes, and that the two error functions do not converge as category set size increases.

The purpose of Experiment 3 was to investigate the discrepancies between the results of Experiments 1 and 2 . Specifically, Experiment 3 tested the prediction that low and high distortions of the empirical and objective prototypes should interact across category set size, a prediction supported by the results of Experiment 1 but not by those of Experiment 2. In Experiment 3, the learning and transfer conditions were identical to those of Experiment 2 , with the following differences: during the transfer phase, subjects classified only old patterns and new distortions of the empirical and objective prototypes. The objective and empirical prototypes were not included as transfer set patterns. It was thought that learning during the transfer phase may have been enhanced by the inclusion of the objective and empirical prototypes. This is a reasonable assumption if it is also assumed that prototypes, more than any other pattern, carry more information about a category.

\section{EXPERIMENT 3}

\section{Method}

Subjects. A total of 117 subjects from the same pool as subjects in Experiments 1 and 2 participated in the experiment and received partial credit toward a course requirement.

Materials and Apparatus. As in Experiments 1 and 2, all stimuli were generated independently for each subject prior to the experimental session. Training sets again included 18 patterns: 9 high 
distortions of one prototype, 6 high distortions of a second, and 3 high distortions of a third. Transfer sets included 21 patterns, 7 from each category. From each category, there were 3 old patterns from the training set, a high distortion of both the objective and empirical prototypes, and a low distortion of both the objective and empirical prototypes. Although the objective and empirical prototypes were necessarily generated for each category, these patterns were not included in the transfer set. The experiment was run on a Terak 8510 microcomputer.

Procedure. Experimental procedures were identical to those described in Experiment 2. During the learning phase, subjects were required to meet the learning criterion of perfect classification performance during one block of trials. After they had reached this criterion, the transfer phase was initiated. If, however, a subject failed to meet the learning criterion after 35 blocks of trials, the experiment was terminated.

\section{Results and Discussion}

Out of 117 subjects participating in the experiment, 19 failed to meet the learning criterion, and analyses were performed on the remaining 98 . For these remaining subjects, the mean number of blocks to criterion was 15.0 .

An analysis of variance was performed treating distortion level as a factor of two levels (high and low), the prototype from which new patterns were derived as a factor with two levels (objective and empirical), and category size as a factor with three levels (three, six, and nine), resulting in a $2 \times 2 \times 3$ factorial design. Mean classification accuracy for all patterns in the transfer phase is shown in Figure 5.

The main effect of category size was significant $[F(2,194)=49.71, M S e=1,914, p<.001]$. Overall, classification of patterns derived from the objective prototype was less accurate than classification of patterns derived from the empirical prototype $[F(1,97)=4.89$, $M S \mathrm{e}=1,464, p<.05]$. This main effect was primarily due to the error differences at category sizes of three and six. Empirical patterns showed no advantage over objective patterns when nine exemplars defined a category during learning. The interaction between category size and prototype approached significance $[F(2,194)=2.73$, $M S \mathrm{e}=1,796, p<.10]$. This was due to the merging of the error rates for objective and empirical distortions as category set size increased. Figure 5 plots mean error rates of transfer patterns as a function of category size. This pattern is similar to the one observed in Experiment 1. As in Experiments 1 and 2, the main effect of distortion was highly significant $[F(1,97)=40.47$, $M S \mathrm{e}=1,740, p<.001]$. Category size also interacted with distortion $[F(2,194)=3.68, M S e=1,680$, $p<.05$ ], reflected by the greater slope of the error rate functions of the highly distorted patterns. No other interactions reached significance.

In a one-factor analysis of variance on classification of performance on old patterns, the main effect of category size was not significant $[F(2,194)=2.52, M S e=1,165$, $p>.10]$.

Apparently, the inclusion of the objective and/or the empirical prototypes had some impact on the classification of new distorted patterns in Experiment 2. In any

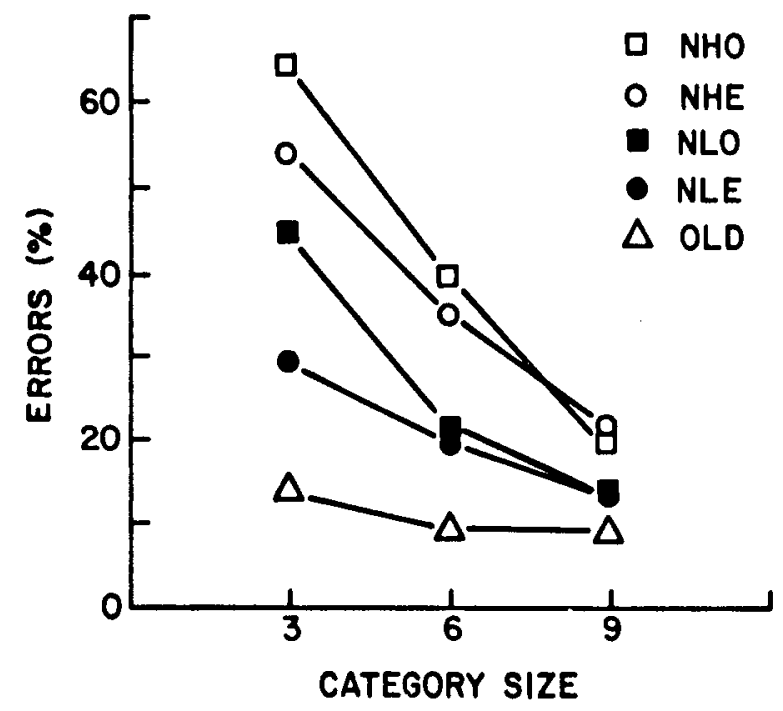

Figure 5. Percent errors for old and new patterns in Experiment 3.

case, it is probably inaccurate to assume that, during the transfer phase, subjects do not acquire any new information about a category and use it in subsequent classifications, even when feedback is not provided. For example, classification of a prototype may influence subsequent classifications of low distortions of that prototype. The prototypes may have more of an impact, or provide the most information about a particular category, because they will be relatively close to other category members presented during the transfer phase. Subjects might use any information acquired during earlier trials in testing, in addition to knowledge about a category acquired during the learning phase in classifying subsequent patterns. There are probably other sources of information available to the subject, in addition to any information acquired during learning, that play a role in classifying patterns during the transfer phase.

\section{GENERAL DISCUSSION}

The strongest point made by these results is purely a methodological one. In many previous studies, it has been shown that objective prototypes are classified more accurately than is any other novel pattern during the transfer phase. In addition, the accuracy with which objective prototypes are classified has sometimes served as a measure of the degree to which an internal prototype, or schema, has been abstracted during learning. These results suggest that the classification of empirical prototypes would serve as a better benchmark with which to evaluate abstraction processes.

The major findings across the three experiments did not completely support our initial predictions. The pattern of convergence of error rates for distortions of the empirical and objective prototypes observed in Experiments 1 and 3 was consistent with our expectations (although this interaction fell short of reaching statistical significance in Experiment 3). However, the lack of convergence 
between error rates on the objective and empirical prototypes themselves in Experiments 1 and 2 is inconsistent with any explanation based on the central limit theorem.

The results are also at first glance counterintuitive to theoretical accounts of the category set size effect, some of which are simply restatements of the central limit theorem. One account (Homa et al., 1973) proposes that during learning an internal prototype is abstracted and then used to classify new patterns in the transfer phase. Classification of novel patterns improves across category set size because the internal, abstracted prototype evolves toward the center of the category with greater learning experience. This results in the internal prototype's moving closer to the objective prototype and distortions of the objective prototype, resulting in more accurate classification. But in Experiments 1 and 2, if the empirical prototype can be regarded as the true center of the category, an interaction between error rates for the empirical and objective prototypes across category set size would be expected.

Intuitively, the same interaction between objective and empirical prototypes would be expected based on Medin and Schaffer's (1978) explanation of the category set size effect. This explanation proposes that with more experience with members of a category, more representations of category exemplars are stored in memory. Usually, classification of novel objects is accomplished by retrieving the most similar exemplar from memory. And if a greater number of exemplars are stored in memory, it becomes more likely that a novel pattern will be more similar to the most similar stored pattern. This explanation can also be inferred from the central limit theorem.

A third explanation may better account for this lack of interaction. This explanation assumes that with relatively little experience with members of a category, classification is mediated by stored exemplar information, and with greater degrees of experience, an abstracted prototype is

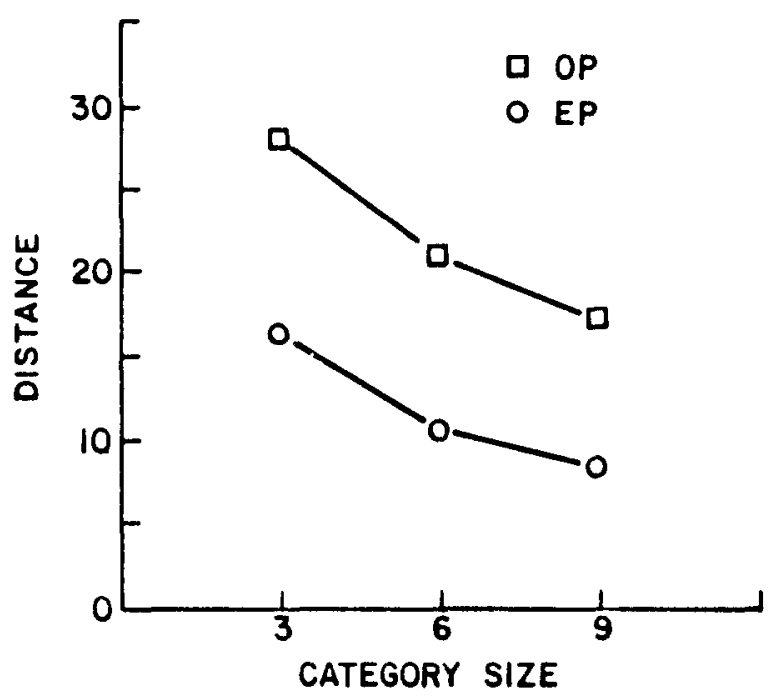

Figure 6. Distances associated with the prototypes after weighting. used more and more in classifying novel category members (e.g., Homa et al., 1981). Classification accuracy for new patterns improves with category set size because the similarity between new patterns and the subjective prototype is greater than the similarity between new patterns and old patterns (i.e., on the average, a distortion of a prototype will be more similar to the prototype than to another distortion of the prototype). One way for this explanation to produce a lack of interaction is shown in Figure 6 . These functions were produced by differentially weighting distances to the most similar old pattern and empirical prototype across category size (see Table 1). That is, a greater weight was given to distances to the empirical prototype at a category set size of nine, and that weighting was decreased as category set size decreased. The weight assigned to distances associated with the most similar old pattern was inversely proportional to the weight associated with the empirical prototype. Specifically, the equation for deriving the distances in Figure 6 was:

$$
[\mathrm{Wi} \times \mathrm{DEPi}]+[(1-\mathrm{Wi}) \times \mathrm{DMSO}],
$$

where DEP is the distance to the empirical prototype, DMSO is the distance to the most similar old pattern, $W$ is the weight associated with distances to the empirical prototype, and i corresponds to category set sizes, 3, 6, or 9 . The weights assigned in this equation to produce the distances in Figure 6 were W3 $=0.4, \mathrm{~W} 6=0.6$, and $\mathrm{W} 9=0.7$.

Figure 6 also reflects one explanation for the rather large impact of category size on error rates for the empirical prototypes in Experiments 1 and 2. By assuming a differential weighting of prototype and exemplar information as category size increases, it is possible to predict that classification of empirical prototypes will be strongly influenced by category size. It is less clear how this effect would be predicted in these experiments if one relied exclusively on prototype or exemplar information.

Finally, it should be noted that sampling a wider range of set sizes most surely would produce a prototype by category size interaction, simply because at some point, with enough patterns in the learning set, the empirical prototype will converge exactly on the objective prototype. However, this fact is precisely why the lack of interaction with the category set sizes sampled in these experiments is not easily explained.

In summary, the results from these experiments present a challenge for classification models. The superiority of the empirical prototypes together with the lack of interaction between category size and prototype runs counter to the statistical fact that the objective and empirical prototypes converge with category size. This finding may require models that assume that with little experience, classification is primarily mediated by exemplar information; and with more exemplar experience, an abstracted prototype becomes more involved in the classification process. 


\section{REFERENCES}

Hintzman, D. L., \& LudLAM, G. (1980). Differential forgetting of prototypes and old instances: Simulation by an exemplar-based classification model. Memory \& Cognition, 4, 378-382.

Homa, D., \& Chambliss, D. (1975). The relative contributions of common and distinctive information on the abstraction from ill-defined categories. Journal of Experimental Psychology: Human Learning \& Memory, 1, 351-359.

Homa, D., Cross, J., Cornell, D., Goldman, D., \& Schwartz, S. (1973). Prototype abstraction and classification of new instances as a function of number of instances defining the prototype. Journal of Experimental Psychology, 101, 116-122.

Homa, D., STERung, S., \& TrePEL, L. (1981). Limitations of exemplarbased generalization and the abstraction of categorical information. Journal of Experimental Psychology: Human Learning \& Memory, 7, 418-439.

Homa, D., \& Vosburgh, R. (1976). Category breadth and the abstrac- tion of prototypical information. Journal of Experimental Psychology: Human Learning \& Memory, 2, 322-330.

MEdin, D. L., \& SCHAFFER, M. M. (1978). Context theory of classification learning. Psychological Review, 3, 207-238.

Posner, M. I., Goldsmith, R., \& Welton, K. E. (1967). Perceived distance and the classification of distorted patterns. Journal of Experimental Psychology, 73, 28-38.

Posner, M. I., \& KEELE, S. W. (1968). On the genesis of abstract ideas. Journal of Experimental Psychology, 77, 353-363.

POSNER, M. I., \& KEELE, S. W. (1970). On the retention of abstract ideas. Journal of Experimental Psychology, 83, 304-308.

Strange, W., Kenney, T., Kessel, F., \& Jenkins, J. (1970). Abstraction over time of prototypes from distortions of random dot patterns. Journal of Experimental Psychology, 3, 508-510.

(Manuscript received May 24, 1985;

revision accepted for publication February 19, 1986.) 